\title{
Note on a thin-shell wormhole in extremal Reissner-Nordström geometry
}

\author{
S. Habib Mazharimousavi* and M. Halilsoy ${ }^{\dagger}$ \\ Department of Physics, Eastern Mediterranean University, \\ Gazimağusa, North Cyprus, Mersin 10 - Turkey.
}

\begin{abstract}
We show that the cold horizon of the extremal Reissner-Nordström can be considered as the throat of a thin-shell wormhole with zero total exotic matter and positive angular pressure. Such a wormhole is physical and stable against radial perturbations provided an appropriate perfect fluid exists at the throat.
\end{abstract}

\section{INTRODUCTION}

The idea of thin-shell wormholes [1] was developed in order to confine the exotic matter sources encountered in wormholes to a narrow hypersurface so that the vast bulk spacetime still possess physical (non-exotic) sources. The induced metric on the throat caries a surface energymomentum of fluid type which satisfies the junction conditions due to Israel's junction formalism [2]. Such a surface fluid is taken to be of the form $S_{\mu}^{\nu}=\operatorname{diag}(-\sigma, p, p)$ in which $\sigma$ stands for the energy-density on the shell and $p$ is the angular pressure. The choice of equation of state for the fluid is problematic since it doesn't satisfy all requirements. It is preferable to have $\sigma>0$ and a reasonable equation of state i.e., $p=p(\sigma)$ so that the energy conditions, at least the null and weak ones are satisfied. In the past, for instance, we had given some models of thin-shell wormholes with non-spherical geometries which had the local energy density negative $(\sigma<0)$ but overall integration of $\sigma$ yields a positive total energy [3]. Restriction of the induced metric to a spherical topology $R \times S^{2}$, unfortunately dashes much of the good aspects in this line of thought. Yet, within the spherical topology it is our belief that there are possible ways to construct physical, i.e. non-exotic thin-shell wormholes 3. In brief, this is the main purpose of the present article. By choosing as our bulk spacetime the extremal Reissner-Nordström (ERN) geometry we show that the energy-density on the shell vanishes with a surface pressure $p=\frac{2}{M}$, which is inversely proportional to the mass (or charge) of the ERN black hole. Vanishing of the energy density $(\sigma=0)$ at the static equilibrium of the thin-shell wormhole is an advantage since to say the least it is better than being negative $(\sigma<0)$. In addition to the energy aspect, stability condition of the thin-shell against linear radial perturbations is of utmost importance. The fact that the shell must be stable against such perturbations for physical viability makes the problem of thin-shell wormholes further difficult to tackle. In this regard we perturb the thin-shell so that the radius becomes a function of the proper time $R(\tau)$. Employing a variable equation of state [4, 5] for the fluid on the shell yields, together with the conservation equation, appropriate forms for $\sigma$ and $p$ which re-

\footnotetext{
* habib.mazhari@emu.edu.tr

$\dagger$ mustafa.halilsoy@emu.edu.tr
}

duce correctly to their static (unperturbed) limits. In 5 where the variable equation of state has been introduced, Varela resolved the anomaly of the instability of the thinshell wormhole in Schwarzschild bulk at $a_{0}=3 M$ i.e., the throat's radius equal to the radius of photon sphere 6 .

As we shall consider the ERN to be the bulk metric we would like to mention that Reissner-Nordström thinshell wormhole has been considered before, first in 8 and later in 9 . In [8] the equation of state was set to $\frac{d p}{d \sigma}=$ $\beta^{2}(\sigma)$ and in their numerical analysis they considered $\beta_{0}^{2}=\beta^{2}\left(\sigma_{0}\right)$. Also in [9] where the equation of state was set to be a Chaplygin gas, $a_{0}=r_{h}$ has been excluded due to the non-physical zone, as they called it in which no solution exists.

\section{THE FORMALISM}

In the standard method of constructing a thin-shell wormhole in spherically symmetric bulk [4, 7] we have

$$
d s_{b u l k}^{2}=-f(r) d t^{2}+\frac{d r^{2}}{f(r)}+r^{2}\left(d \theta^{2}+\sin ^{2} \theta d \phi^{2}\right)
$$

with induced metric at the throat

$$
d s_{\text {throat }}^{2}=-d \tau^{2}+R^{2}(\tau)\left(d \theta^{2}+\sin ^{2} \theta d \phi^{2}\right)
$$

in which $\tau$ stands for the proper time. We find the energy density and angular pressure from $S_{\mu}^{\nu}=\operatorname{diag}(-\sigma, p, p)$ of the perfect fluid presented at the throat given by $(8 \pi G=$ 1)

$$
\sigma=-4\left(\frac{\sqrt{f(R)+\dot{R}^{2}}}{R(\tau)}\right)
$$

and

$$
p=2\left(\frac{2 \ddot{R}(\tau)+f^{\prime}(R)}{2 \sqrt{f(R)+\dot{R}^{2}}}+\frac{\sqrt{f(R)+\dot{R}^{2}}}{R(\tau)}\right) .
$$

Note that we use the notation such that a dot stands for $\frac{d}{d \tau}$ and a prime implies $\frac{d}{d R}$. In static condition one finds

$$
\sigma_{0}=-\frac{4 \sqrt{f\left(R_{0}\right)}}{R_{0}}
$$


and

$$
p_{0}=2\left(\frac{f^{\prime}\left(R_{0}\right)}{2 \sqrt{f\left(R_{0}\right)}}+\frac{\sqrt{f\left(R_{0}\right)}}{R_{0}}\right) .
$$

Next, if we assume that the bulk spacetime is a black hole with the event horizon at $r=r_{h}$, naturally one must impose $R_{0}>r_{h}$. This is due to the fact that $p_{0}$ is diverging at the horizon which is not acceptable for a physical wormhole. Note that $R_{0}=r_{h}$ is also excluded for the same reason.

\section{A. Thin-shell wormhole in extremal black hole bulks}

Here we consider the bulk spacetime to be an extremal black hole such that $f\left(r_{h}\right)=0$ and is same for its first derivative i.e., $f^{\prime}\left(r_{h}\right)=0$. More precisely we set $f(r)=$ $U(r)^{2}$ with $U\left(r_{h}\right)=0$ satisfying $U(r)>0$ for $r>r_{h}$. In this configuration if we set $R_{0}=r_{h}$ we find $\sigma_{0}=0$ and

$$
p_{0}=2 U^{\prime}\left(r_{h}\right)
$$

which is positive and finite. The well known extremal Reissner-Nordström (ERN) black hole is a candidate for this arrangement. The metric of ERN is given by $f(r)=$ $\left(1-\frac{M}{r}\right)^{2}$ with a double root event horizon located at $r_{h}=M(=Q=$ the charge). Hence the angular pressure of the thin-shell wormhole located at $R_{0}=M$ is given by $p_{0}=\frac{2}{M}$ with vanishing energy density $\sigma_{0}=0$. For the static setting in the frame of comoving observer located on the throat with proper time $\tau$, the thin-shell is made of a perfect fluid with the energy density zero and a positive angular pressure $p_{0}=\frac{2}{M}$ which is inversely proportional to the mass of the ERN black hole.

\section{B. Stability}

Our next concern is to investigate the stability of the thin-shell wormhole with a throat located at the horizon of an extremal black hole, specifically, ERN. We perturb the thin-shell wormhole radially and due to that the energy density and pressure become dynamic given by

$$
\sigma=-4\left(\frac{\sqrt{U^{2}+\dot{R}^{2}}}{R(\tau)}\right)
$$

and

$$
p=2\left(\frac{\ddot{R}(\tau)+U U^{\prime}}{\sqrt{U^{2}+\dot{R}^{2}}}+\frac{\sqrt{U^{2}+\dot{R}^{2}}}{R(\tau)}\right)
$$

where $R(\tau)$ is the throat radius after the perturbation. One can show that $\sigma$ and $p$ satisfy the energy conservation law given by

$$
\frac{d \sigma}{d \tau}=-\frac{2 \dot{R}}{R}(p+\sigma)
$$

or equivalently

$$
\frac{d \sigma}{d R}=-\frac{2}{R}(p+\sigma)
$$

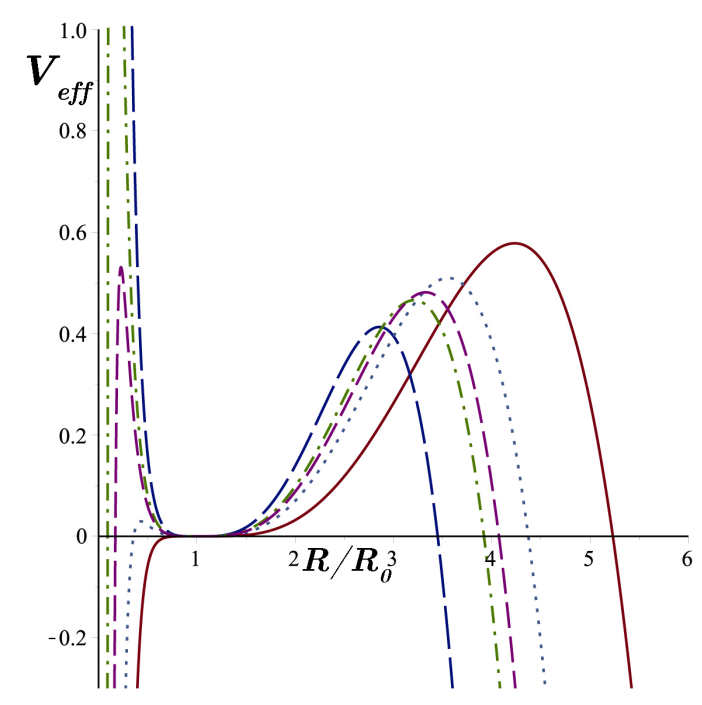

FIG. 1: Effective potential $V_{\text {eff }}$ vs $\frac{R}{R_{0}}$ for $\omega=0, \frac{1}{10}, \frac{1}{8}, \frac{1}{6}$ and $\frac{1}{4}$ in long-dash, dot-dash,dash, dot and solid respectively.

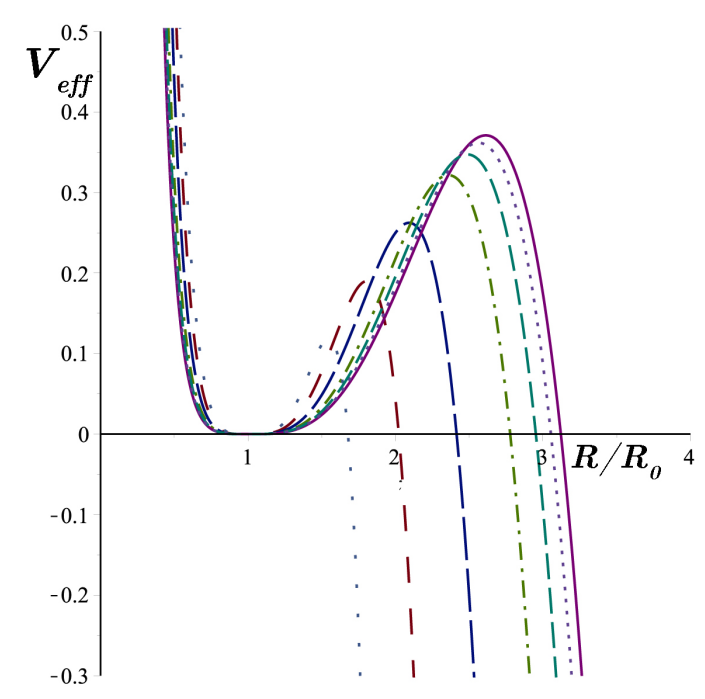

FIG. 2: Effective potential $V_{\text {eff }}$ vs $\frac{R}{R_{0}}$ for $\omega=-\frac{1}{10},-\frac{1}{8},-\frac{1}{6},-\frac{1}{4},-\frac{1}{2},-1$ and -2 in solid, dot, dash, dot-dash, long-dash, space-dash and space-dot respectively. In the case of $\omega=-1$ one must use the limit of the potential.

In addition to that one also finds from (3) that

$$
\dot{R}^{2}+\left(U^{2}-\left(\frac{\sigma R}{4}\right)^{2}\right)=0
$$

which is the equation of motion of the shell with an effective potential $V_{e f f}=U^{2}-\left(\frac{\sigma R}{4}\right)^{2}$. The last two equations are coupled such that one can find a solution for 
$\sigma$ in (11) and consequently from (12) we find the behaviour of $R$ with respect to the proper time $\tau$. To find $\sigma$ from (11) we must assume an equation of state of the form $p=p(\sigma)$ which satisfies the boundary conditions i.e., $p\left(\sigma_{0}=0\right)=p_{0}$ at $R=R_{0}$. After [4, 5] we choose the following equation of state for the perfect fluid at the location of the throat

$$
p=\omega \sigma+\frac{2(2 \omega-1)}{R_{0}}\left(\frac{R}{R_{0}}-1\right)+\frac{2}{R_{0}}
$$

in which $\omega$ is a constant. Hence, the conservation equation admits

$$
\sigma=\frac{4\left(\frac{R}{R_{0}}-3\right)-4 \omega(2 \omega+1)\left(\frac{R}{R_{0}}-1\right)+8\left(\frac{R_{0}}{R}\right)^{2(\omega+1)}}{R_{0}(\omega+1)(3+2 \omega)}
$$

in which the integration constant has been set such that $\sigma\left(R_{0}\right)=0$ and naturally $\omega=-\frac{3}{2}$ must be excluded. The case for $\omega=-1$ can be checked to exist from the L'Hospital's limiting procedure. Considering this energy density in the effective potential of the one-dimensional equation of motion (12) we find

$$
\begin{aligned}
& V_{\text {eff }}(R)=\left(1-\frac{R_{0}}{R}\right)^{2}- \\
& \left(\frac{\omega-1}{\omega+1} \frac{R}{R_{0}}+\frac{2\left(\frac{R_{0}}{R}\right)^{2 \omega+1}}{(\omega+1)(3+2 \omega)}-\frac{2 \omega-1}{3+2 \omega}\left(\frac{R}{R_{0}}\right)^{2}\right)^{2} .
\end{aligned}
$$

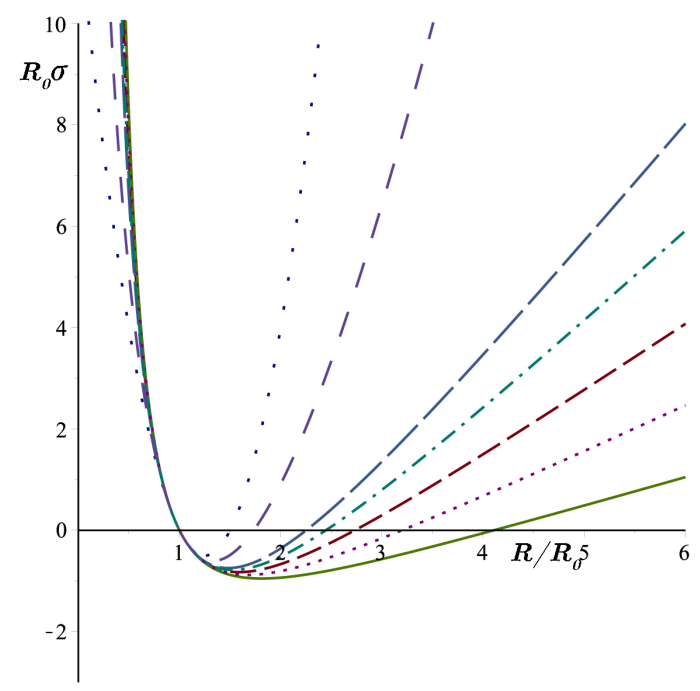

FIG. 3: $R_{0} \sigma$ vs $\frac{R}{R_{0}}$ for $\omega=\frac{1}{4}, \frac{1}{8}, 0,-\frac{1}{8},-\frac{1}{4},-1$ and -2 in solid, dot, dash, dot-dash,long-dash, space-dash and space-dot respectively.

In Figs. 1 and 2 we plot $V_{\text {eff }}(R)$ versus $\frac{R}{R_{0}}$ for various values of $\omega<\frac{1}{4}$. Admitting a relative minimum at $\frac{R}{R_{0}}=1$ implies that the thin-shell wormhole is stable against radial perturbations. As is clear from the Figs. 1 and 2, in

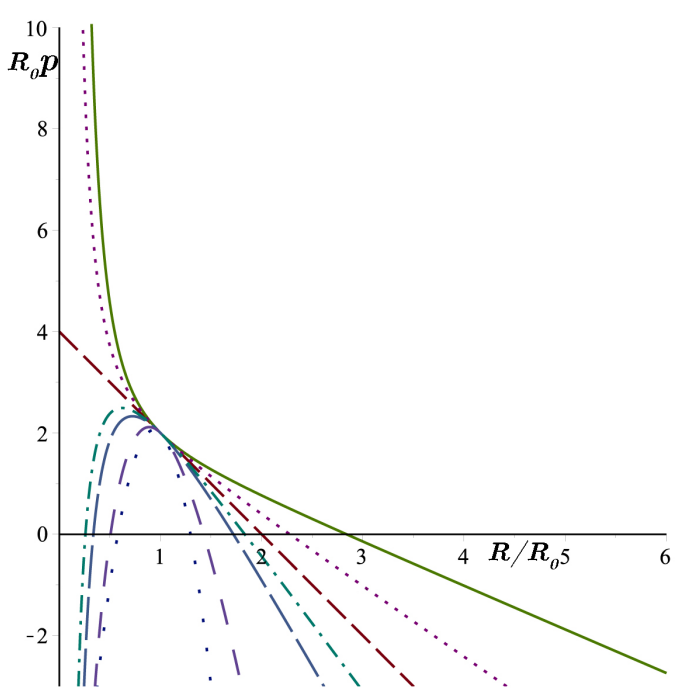

FIG. 4: $R_{0} p$ vs $\frac{R}{R_{0}}$ for $\omega=\frac{1}{4}, \frac{1}{8}, 0,-\frac{1}{8},-\frac{1}{4},-1$ and -2 in solid, dot, dash, dot-dash,long-dash, space-dash and space-dot respectively.

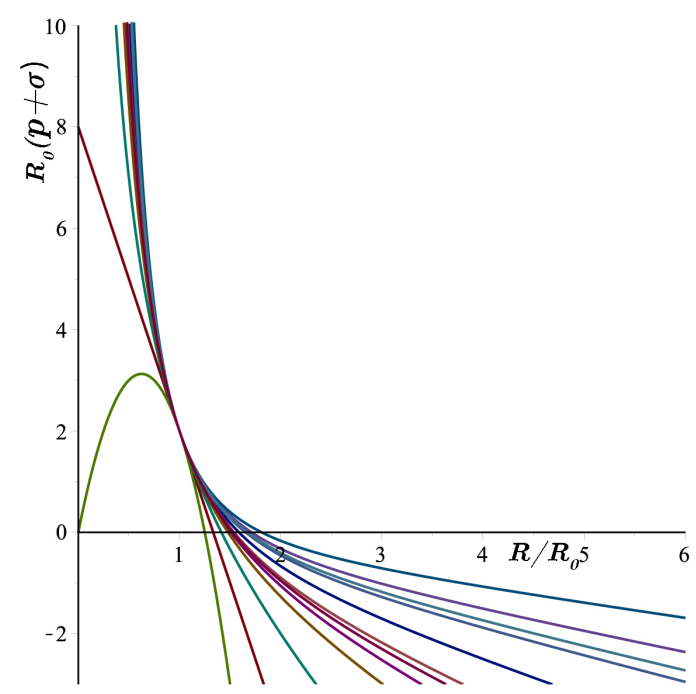

FIG. 5: $R_{0}(p+\sigma)$ vs $\frac{R}{R_{0}}$ for

$\omega=\frac{1}{4}, \frac{1}{6}, \frac{1}{8}, \frac{1}{10}, 0,-\frac{1}{10},-\frac{1}{8},-\frac{1}{4},-\frac{1}{2},-1$ and -2 from the right to
the left respectively.

the neighborhood of $\omega=0$ the stability is stronger as the heights of the barriers are getting higher. Furthermore, for strong perturbation even with $\omega<\frac{1}{4}$ the thin-shell may be unstable while for positive $\omega$ both possibility i.e., collapse and evaporation are likely to happen whereas for $\omega<0$ the collapse can not take place. We also observe that for $\omega \geq \frac{1}{4}$ the potential does not admit a minimum at the location of the throat $R=R_{0}$ and therefore the thin-shell wormhole becomes unstable. In Figs. 3 and 4 we plot $R_{0} \sigma$ and $R_{0} p$ in terms of $\frac{R}{R_{0}}$ for some values of $\omega$ which have been used in Figs. 1 and 2. In Fig. 5 we plot $R_{0}(\sigma+p)$ versus $\frac{R}{R_{0}}$ for various $\omega$ 's. Clearly in the vicinity of $\frac{R}{R_{0}}=1, \sigma+p$ is positive, indicating that the 
energy conditions are satisfied, at least for $\frac{R}{R_{0}} \leq 1$.

\section{CONCLUSION}

We constructed thin-shell wormholes in extremal Reissner-Nordström spacetime with its static throat $R=$ $R_{0}$ located at the double root horizon i.e., $r_{h}=R_{0}=$ $M=Q$. We have shown that at static equilibrium the perfect fluid presented at the throat possesses $\sigma_{0}=0$ and $p_{0}=\frac{2}{M}$ which clearly satisfy the energy conditions. Within the equation of energy conservation (11) we found the exact forms of the energy density and angular pressure when the thin-shell is perturbed radially. Also we found a one-dimensional equation of motion for the throat after the perturbation whose onedimensional effective potential admits (for specific values of $\omega$ ) a local minimum at the location of the static radius. This is an indication for the stability of the thin-shell against a radial perturbation. The equation of state which we adopted on the shell is of the form $p=\omega \sigma+\frac{2(2 \omega-1)}{R_{0}}\left(\frac{R}{R_{0}}-1\right)+\frac{2}{R_{0}}$, in which $\omega$ is a constant parameter. The fact that the role of the parameter $\omega$ is critical both in stability and energy conditions can be seen from the plotted curves. The perturbation equation for the radius of the shell satisfies an equation $\dot{R}^{2}+V_{\text {eff }}=0$, where $\dot{R}=\frac{d R}{d \tau}$ and $V_{\text {eff }}$ stands for an effective potential i.e., Eq. (12). The plot of the potential $V_{\text {eff }}$ versus $\frac{R}{R_{0}}$ yields potential well in the vicinity of $\frac{R}{R_{0}}=1$ which renders stable configurations for finely tuned parameter $\omega$. We plot also $\sigma(R)$ which yields positive values in certain regions of $R$. Further to $\sigma$, we also investigate the combination $\sigma+p$, which possesses a positive domain and therefore satisfies the energy conditions. Beyond certain range for the parameter $\omega$ we observe that the shell becomes unstable. As a result there are two possibilities: either the shell collapses to the center or it expands indefinitely. As a final point let us remark that what has been done about the cold, i.e., zero Hawking temperature, ERN black hole, are also valid for other cold / ultracold extremal black holes.
[1] M. Visser, Phys. Rev. D 39, 3182 (1989).

[2] G. Darmois (1927) Mémorial de Sciences Mathématiques, Fascicule XXV, "Les equations de la gravitation einsteinienne", Chapitre V.

W. Israel "Thin shells in general relativity". Il. Nuovo Cim. 66, 1 (1966).

[3] S. H. Mazharimousavi and M. Halilsoy, Eur. Phys. J. C 75, 540 (2015);

S. H. Mazharimousavi and M. Halilsoy, Eur. Phys. J. C 75, $271(2015)$;

S. H. Mazharimousavi and M. Halilsoy, Eur. Phys. J. C 75, 81 (2015);
S. H. Mazharimousavi and M. Halilsoy, Eur. Phys. J. C 74, 3067 (2014).

[4] N. M. Garcia, F. S. N. Lobo and M. Visser, Phys. Rev. D 86, 044026 (2012).

[5] V. Varela, Phys. Rev. D 92, 044002 (2015).

[6] E. Poisson and M. Visser, Phys. Rev. D 52, 7318 (1995); F. S. N. Lobo and P. Crawford, Classical Quantum Gravity 21, 391 (2004);

G. A. S. Dias and J. P. S. Lemos, Phys. Rev. D 82, 084023 (2010).

[7] E. F. Eiroa, Phys. Rev. D 78, 024018 (2008).

[8] E. F. Eiroa and G. E. Romero, Gen. Relativ. Gravit. 36, 651 (2004).

[9] M. Sharif and M. Azam, Eur. Phys. J. C 73, 2554 (2013). 